\title{
Vitamina C e a relação com a imunidade e como Agente Preventivo da COVID- 19
}

\section{(Sars -Cov2)}

Vitamin C and the relationship with immunity and as a Preventive Agent for COVID- 19 (Sars -

$\operatorname{Cov} 2)$

La vitamina c y la relación con la inmunidad y como Agente Preventivo del COVID-19 (Sars -Cov2)

Recebido: 31/03/2021 | Revisado: 10/04/2021 | Aceito: 14/04/2021 | Publicado: 26/04/2021

Michelle Nogueira de Jesus
ORCID: https://orcid.org/0000-0001-8670-4268
E-mail: michellenoguei18@gmail.com
Aline Cristine Ferreira Felipe Rocha
ORCID: https://orcid.org/0000-0001-8737-9348
E-mail:alinefelipe.nutri@ @mail.com
Stéphani Borges Campos
Instituto Federal de Educação, Ciência e Tederal de Educaço, Ciência e Tecnologia Goiano, Brasil
ORCID: https://orcid.org/0000-0001-8343-5058
E-mail:stephani.borges@estudante.ifgoiano.edu.br
Tâmara Fabíola Vieira Santana
ORCID: https://orcid.org/0000-0002-8151-151X
Instituto Federal de Educação, Ciência e Tecnologia Goiano, Brasil
E-mail:ciuzefab@hotmail.com
Geovana Rocha Plácido
Instituto Federal de Educação, Ciência e Tecnologia Goiano, Brasil
ORCID: https://orcid.org/0000-0002-3028-7191
Instituto Federal de Educação, Ciência e Tecnologia Goiano, Brasil
E-mail: geovana.placido@ifgoiano.edu.br

\section{Resumo}

A vitamina $\mathrm{C}$ também denominada ácido ascórbico é um antioxidante, cuja função está relacionada a melhora do sistema imune, proteção contra doenças cardiovasculares, auxilia no combate ao estresse, além de proteção de unhas, cabelos e olhos. Sabendo de sua importância para imunidade e levando em consideração sua atuação como agente preventivo de doenças, o objetivo desse trabalho consistiu em realizar um levantamento bibliográfico sobre a atuação da vitamina $\mathrm{C}$ em relação a imunidade e como ela age contra a doença COVID - 19 também conhecida como Corona vírus, apresentando a importância dessa vitamina e seus mecanismos de ação no organismo humano. A partir da revisão realizada, notou-se que a vitamina $\mathrm{C}$ é essencial para o sistema imunológico e que a falta dessa leva a uma doença chamada de escorbuto, além disso notou-se que o ácido ascórbico se encontra modo natural em alguns alimentos, principalmente cítricos, e o mesmo ainda é adicionado em outros suplementos. Com relação a vitamina $\mathrm{C}$ como agente preventivo da COVID-19 mostrou que pacientes tratados com alta dose tiveram melhora significativas tanto em adultos quanto crianças. Sendo assim e considerando os estudos realizados conclui-se que a vitamina $\mathrm{C}$ possui que a vitamina $\mathrm{C}$ melhora o sistema imunológico estando associada na regulação de uma vida saudável. Palavras-chave: Ácido ascórbico; Sistema imune; Corona vírus.

\begin{abstract}
Vitamin $\mathrm{C}$ also called ascorbic acid is an antioxidant, whose function is related to the improvement of the immune system, protection against cardiovascular diseases, helps in combating stress, in addition to protection of nails, hair and eyes. Knowing its importance for immunity and taking into account its role as a disease preventive agent, the objective of this work was to carry out a bibliographic survey on the role of vitamin $\mathrm{C}$ in relation to immunity and how it works against COVID - 19 disease, also known like Corona virus, showing the importance of this vitamin and its mechanisms of action in the human body. From the review carried out, it was noted that vitamin $\mathrm{C}$ is essential for the immune system and that the lack of it leads to a disease called scurvy, in addition it was noted that ascorbic acid is found naturally in some foods, especially citrus, and it is still added in other supplements. Regarding vitamin $\mathrm{C}$ as a preventive agent, COVID-19 showed that patients treated with a high dose had significant improvements in both adults and children. Therefore, considering the studies carried out, it is concluded that vitamin $\mathrm{C}$ has that vitamin $\mathrm{C}$ improves the immune system and is associated with the regulation of a healthy life.
\end{abstract}

Keywords: Ascorbic acid; Immune system; Corona virus. 


\section{Resumen}

La vitamin C también llamada ácido ascórbico es un antioxidante, cuya función está relacionada con la mejora del sistema inmunológico, protección frente a enfermedades cardiovasculares, ayuda a combatir el estrés, además de protección de uñas, cabello y ojos. Conociendo su importancia para la inmunidad y teniendo en cuenta su papel como agente preventivo de enfermedades, el objetivo de este trabajo fue realizar un relevamiento bibliográfico sobre el papel de la vitamina $\mathrm{C}$ en relación con la inmunidad y cómo actúa contra la enfermedad COVID-19, también conocido como virus Corona, mostrando la importancia de esta vitamina y sus mecanismos de acción en el cuerpo humano. De la revisión realizada, se observó que la vitamina $C$ es esencial para el sistema inmunológico y que la falta de ella conduce a una enfermedad llamada escorbuto, además se observó que el ácido ascórbico se encuentra de forma natural en algunos alimentos, especialmente los cítricos, y todavía se agrega en otros suplementos. Con respecto a la vitamina $\mathrm{C}$ como agente preventivo, COVID-19 mostró que los pacientes tratados con dosis altas tuvieron mejoras significativas tanto en adultos como en niños. Por tanto, considerando los estudios realizados, se concluye que la vitamina $\mathrm{C}$ posee que la vitamina $\mathrm{C}$ mejora el sistema inmunológico y está asociada a la regulación de una vida sana.
\end{abstract}

Palabras clave: Ácido ascórbico; Sistema inmunológico; Virus corona.

\title{
1. Introdução
}

O isolamento social, devido a pandemia mundial provocada pela COVID-19, levou pessoas a buscarem uma alimentação adequada (Campos, 2020). Foi o que demonstrou uma pesquisa realizada de forma online, onde houve aumento na procura por alimentos e nutrientes que atuem no sistema imunológico, sendo que antes da pandemia, o consumo de nutrientes era de $56,16 \%$, evoluindo, para $71,63 \%$, e constatou ainda, que dentre os mais consumidos estavam vitaminas C e D (Mendes, et al., 2020).

A alimentação saudável é definida como aquela que atende todas as exigências do corpo, e que possui a quantidade adequada de macronutrientes (proteínas, carboidratos e lipídios) e micronutrientes (vitaminas e minerais) que auxiliam no funcionamento e nas necessidades fisiológicas do organismo (Dutra, et al., 2020).

A COVID-19 causada pelo coronavírus SARS-CoV-2 (Li, 2016) é transmitida, através de gotículas de saliva, descargas respiratórias ou contato com objetos contaminados (Rothan \& Byrareddy, 2020). Os sintomas mais comuns da doença incluem febre, tosse, dispneia, mialgia, dor de cabeça e diarreia (Lai, et al., 2019).

Lima et al., (2020), relatam que devido ao efeito antioxidante da vitamina $\mathrm{C}$ e sua capacidade de atuar reduzindo infecções do trato respiratório inferior, o seu uso pode ser uma terapia eficaz para pacientes com COVID-19. Em outro estudo sobre o papel da vitamina $C$ na prevenção da pneumonia, os autores concluíram que, mesmo com este composto já sendo utilizado para tal finalidade, as evidências para esta situação não se sustentam e algum efeito pode ser positivo para aqueles cuja ingestão diária esteja dentro dos limites recomendados que é de até $2 \mathrm{~g}$ ao dia (Bonfim, et al., 2020).

A vitamina C é solúvel em água, possui duas formas bioativas, L- ácido ascórbico e ácido desidroascórbico, sendo espontaneamente encontrada em alimentos, especialmente em frutas e vegetais (Pénicaud, et al, 2010; Travica, et al., 2017).

Doadora de elétrons e, portanto, um agente redutor, a vitamina $\mathrm{C}$ possui ação antioxidante, sendo que todas as ações fisiológicas e bioquímicas conhecidas da vitamina, são devidas a essa característica (Dennis \& Wittings, 2017). A vitamina C está presente em maior proporção espécies vegetais, sendo presente em alimentos de origem animal, porém em menores concentrações (Spinola, 2011).

A alimentação e nutrição possui estreita relação com o sistema imune, pois há interação entre a ingestão adequada de nutrientes, o aumento no estresse oxidativo e a ocorrência de processos infecciosos com depleção imunitária, uma ingestão adequada de alimentos com nutrientes específicos como a vitamina $\mathrm{C}$ auxiliam funções das células de defesa do corpo e contribui para melhora na qualidade de vida (Biasebetti, Rodrigues \& Mazur, 2018).

A deficiência de nutrientes é um dos grandes problemas atuais da população, Siqueira et al., (2020) avaliaram, o custo-benefício dos nutrientes dos alimentos consumidos no Brasil, e verificou que a vitamina $C$, foi o nutriente mais barato, e 
acessível, a ser inserido na dieta brasileira, sendo que com $\mathrm{R} \$ 0,01$ é possível suprir $30 \%$ das necessidades diárias desse nutriente, e verificou que a falta desses alimentos na alimentação está mais relacionada a decisão de compra.

Barazzoni et al., (2020) relatam que uma má nutrição ocasionada pela carência nutricional e a fome foram associadas a gravidade da COVID-19 e estão relacionadas com a mortalidade na população mais jovem. Sendo assim o presente estudo objetivou realizar um levantamento bibliográfico entre a relação da vitamina $\mathrm{C}$ com o sistema imune e como agente preventivo da COVID - 19.

\section{Metodologia}

Esse trabalho é de natureza qualitativa pois as informações apresentadas são advindas de um levantamento bibliográfico, facilitando a compreensão do estudo (Pereira et al, 2018). A pesquisa realizada abrange os principais trabalhos publicados sobre a vitamina $\mathrm{C}$ como agente preventivo da COVID-19 e adjuvante da imunidade, publicados nos dez últimos anos.

Os artigos foram obtidos dos seguintes bancos de dados: Web of Science, Scielo, ScienceDirect, MEDLINE/PubMed, incluindo publicações nacionais e internacionais, correspondente a estudos relacionados a vitaminas $\mathrm{C}$ e saúde. Os critérios de inclusão foram artigos publicados na íntegra, gratuitos e online publicados até o presente momento, além disso foram excluídos os artigos que não discorriam acerca do tema proposto.

\section{Revisão Bibliográfica}

\subsection{Recomendação e Fonte Alimentar}

A dose de ingestão recomendada de vitamina C para homens adultos, é $90 \mathrm{mg} /$ dia e para mulheres adultas $75 \mathrm{mg} / \mathrm{dia}$, segundo Recommended Dietary Allowances (RDA) (RDA, 2000). Sendo que, para crianças de 0 a 12 meses a ingestão dessa vitamina é de $20 \mathrm{mg} / \mathrm{dia}$, segundo a Autoridade Europeia para Segurança dos Alimentos (EFSA, 2013).

As principais fontes da vitamina C são, frutas cítricas como limão e laranja, além de acerola, caju, goiaba, repolho, brócolis, couve-flor, groselhas pretas, pimentão doce, salsa, batata, batata doce, couves de bruxelas, morango e manga e em alimentos de origem animal como fígado, leite e carne, mas para estes em quantidades menores (Cavalari \& Sanches, 2018).

Dentre os alimentos relacionados na Tabela de Composição de Alimentos Brasileira, a acerola é a fruta com maior concentração de vitamina C, pois em 100g, 941,4 mg são dessa vitamina (NEPA, 2011).

\subsection{Deficiência de vitamina $\mathrm{C}$}

As vitaminas são utilizadas na precaução de tratamento de déficit nutricional e terapia de doenças não relacionadas à deficiência. (Fuchs \& Wannm, 2010). O principal objetivo em manter uma ingestão adequada de ácido ascórbico é bloquear o escorbuto e fornecer proteção antioxidante, sendo que o escorbuto é a principal doença causada pela falta de ácido ascórbico (Mcevoyg, 2011).

Os seres humanos possuem necessidade absoluta de vitamina $\mathrm{C}$ (ascorbato) como parte de sua dieta, e a deficiência devido à ingestão inadequada está associada a uma infinidade de sintomas, refletindo as diversas funções atribuídas à vitamina (Grosso, 2013). Estruturas colagenosas são primeiramente acometidas e ocorre desenvolvimento de lesões nos ossos e vasos sanguíneos, a administração de ácido ascórbico transforma totalmente os sinais apresentados, assim como os sintomas que surgem em decorrência da deficiência dessa vitamina. (Mcevoyg, 2011).

A vitamina $\mathrm{C}$, possui diversas características que a tornam interessante para prevenir e curar infecções virais. A ação antiviral do ácido ascórbico já é conhecida há, pelo menos, 80 anos. O seu uso como medicamento contra doenças infecciosas 
também é bastante relatado, distintos mecanismos fazem parte da ação antiviral da vitamina C, incluindo as imunomodulatórias (Allegra, et al., 2020).

\subsubsection{Deficiência de Vitamina C como fator de risco para O COVIV-19}

Allegra et al., (2020), propõe que a vitamina C, reduza a duração da gripe comum, mas os efeitos contra a COVID-19 não são conhecidos. Uma análise de 12 estudos com 1.766 pacientes internados em UTI mostrou que a vitamina C reduziu a duração da internação em $8 \%$. Em outra análise, oito estudos demonstraram que a vitamina C reduziu o período de ventilação mecânica em pacientes que necessitaram desta intervenção.

Há evidências que as concentrações de vitamina C, reduzem drasticamente em pacientes acometidos por doença severa, e considera- se que a vitamina $\mathrm{C}$ é capaz de reduzir a tempestade de citocinas via mecanismos desconhecidos, sendo que citocina é o nome geral dado a qualquer proteína que é secretada por células e que afeta o comportamento das células vizinhas portadoras de receptores adequados (Murphy, 2015).

Em 26 de junho de 2020, existiam 25 testes clínicos registrados para testar o efeito da suplementação de vitamina C em pacientes infectados pelo SARS-CoV-2, alguns desses protocolos são testes controlados com a monoterapia com vitamina C para tratamento da COVID-19, enquanto outros protocolos testam a vitamina $\mathrm{C}$ concomitante a outros medicamentos, tanto no tratamento como na prevenção da doença (Allegra, et al., 2020).

Um teste clínico randomizado está analisando a eficácia e segurança do uso da vitamina C contra a pneumonia associada à COVID-19. Há necessidade urgente de se examinar os usos da vitamina C na pré-infecção e na pós-infecção, assim como nos diferentes estágios da doença. Como tudo na vida, a resposta e o equilíbrio, onde não falte a vitamina C, e nem haja excesso desnecessário (Allegra, et al., 2020).

\subsection{Mecanismo de ação da vitamina $C$}

A vitamina $\mathrm{C}$ oferece suporte a várias funções celulares, incluindo a modificação da suscetibilidade a várias infecções virais e influenciando a inflamação, um dos principais direcionadores desse interesse é que os leucócitos acumulam a vitamina em altas concentrações intracelulares, sinalizando um importante papel para ela nessas células (Ang, 2018).

O intestino tem a capacidade em absorver o ácido ascórbico em aproximadamente $1200 \mathrm{mg} / 24 \mathrm{~h}$. Quando o fornecimento de ácido ascórbico aumenta muito, a absorção diminui, passando de 49,5\% para uma dose oral de 1,5 g, a 16,1\%, para uma dose de $12 \mathrm{~g}$ (Guilland, 1995).

Ang (2018) relata que absorção do ácido ascórbico ocorre no jejuno e no íleo, que são porções distais do intestino delgado, sendo para isso, necessário que contenha de sódio na luz intestinal). Ainda segundo o autor, as concentrações de ascorbato intracelular em linfócitos circulantes, monócitos e neutrófilos foram relatadas como sendo 3,5, 3 e 1,5 mM, respectivamente, quando os níveis plasmáticos são de pelo menos $50 \mu \mathrm{M}$, refletindo o estado em indivíduos saudáveis que consomem $\geq 100 \mathrm{mg}$ de ascorbato diariamente (Ang, 2018).

\subsection{Mecanismo de ação do COVID -19}

A COVID-19 ocasionada pelo coronavírus SARS-CoV-2, que faz parte de um grupo de coronavírus que inclui quatro gêneros principais: Alfacoronavírus, Betacoronavírus, Gamacoronavirus e Deltacoronavirus (Li, 2016). O SARS-CoV-2 é classificado como um Betacoronavirus (Lu, 2020), e apresenta-se clinicamente de forma leve, moderada, grave ou crítica (Wang, et al., 2020). 
O mecanismo de virulência do coronavírus, inclui a enzima conversora de angiotensina 2 (ACE2), que é o receptor crucial para garantir que o SARS-CoV-2 entre na célula hospedeira (Lan, et al., 2020). Consequentemente, os órgãos que expressam ACE2 têm maior probabilidade de sofrer danos relacionados à SARS-CoV-2, como pulmões e trato gastrointestinal.

A COVID-19 carece de terapia específica e definida, e as intervenções dependem inteiramente da apresentação. As formas assintomáticas e levemente sintomáticas precisam de pouca ou nenhuma intervenção médica, mas devem ser monitoradas e necessárias para isolamento (Scavone, et al., 2020).

O manejo clínico de casos moderados, graves ou críticos de COVID-19 gira principalmente em torno de tratamentos sintomáticos e de suporte, incluindo hospitalização ou terapia intensiva (Wang et al, 2020). Para o tratamento sintomático da doença, recomenda-se o uso de acetaminofeno em vez do ibuprofeno como agente antipirético (Day, 2020). Os antibióticos empíricos são a escolha preferida para a prevenção de infecção secundária da COVID-19 (Huang, et al., 2020).

Dependendo da gravidade da dispneia e do nível de saturação do paciente, a oxigenoterapia geral, o oxigênio de alto fluxo, a ventilação não invasiva e a ventilação mecânica invasiva são escolhidas para garantir a segurança da vida dos pacientes (Zeng, et al., 2020).

\subsection{Vitamina C e Imunidade}

A reação de defesa do organismo é representada pelo sistema imunológico, composto por uma complexa rede dispersa pelo organismo (Biasebetti, Rodrigues \& Mazur, 2018). O sistema imune possui resposta inata e adaptativa, que são ações específicas do antígeno (Calder, et. al., 2020).

O conjunto de respostas inatas é construído por barreiras que auxiliam a prevenir o acesso de patógenos quando o organismo sofre ameaças, por meio de processos inflamatórios, onde agem rapidamente reparando a inflamação e os prejuízos causados e após a resposta inata a resposta adaptativa é acionada, para desenvolver a resposta imunológica (Calder, et. al., 2020).

A vitamina $\mathrm{C}$ melhora a quimiotaxia dos neutrófilos, a fagocitose e a depuração microbiana (Carr \& Maggini, 2017). Além de promover aumento de células T, células natural killer e modular suas funções (Van Gorkom, et al., 2018). Conhecida por possui efeitos imunoestimulantes, propriedades antioxidantes, anti-inflamatórias, antivirais e efeito antimutagênico (Dennis \& Witting, 2017).

Estudos de meta-análise demonstraram que a vitamina $\mathrm{C}$, pode reduzir o tempo de permanência do paciente em Unidades de Terapia Intensiva (UTI) e encurtar, o tempo de duração da ventilação mecânica (Hemilä \& Chalker, 2019; Hemilä \& Chalker, 2020).

\subsection{Vitamina C e COVID 19}

A vitamina $\mathrm{C}$ é reconhecida como um dos principais componentes da dieta, por possuir efeitos imunomoduladores e ocasionar benefícios em doenças infecciosas, e por possuir uma ação considerada protetora contra infecções respiratórias torna-o um alvo de interesse na COVID-19 (Shakoor, et al., 2021).

Durante o período da infecção ocasionada pela COVID-19, ocorre um aumento de liberação de citocinas à medida que a infecção progride, e a vitamina $\mathrm{C}$ foi sugerida como um contra-ataque a essa ação, por ser capaz de reduzir níveis de citocinas pró-inflamatórias, incluindo TNF- $\alpha$ e aumentar as citocinas antiinflamatórias (IL-10), que funcionam como um feedback negativo com a IL-6 e controla a inflamação crítica na COVID-19 (Zhou \& Xu, 2020).

Shakoor et al., 2021 considera, a suplementação de vitamina C uma opção sensata em indivíduos com deficiência de micronutrientes que estão em risco de infecção por COVID-19 para auxiliar na prevenção e suporte de respostas imunológicas. Além disso, o tratamento com vitamina C, melhora a sobrevivência de pessoas estressadas (Marik, 2020). 
Durante o surto de SARRS-CoV-1 em 2003, o uso de vitamina C, foi sugerido como um tratamento não especifico para infecções virais graves do trato respiratório (Arabi, Fowler \& Hayden, 2020). Segundo Gombart, Pierre e Maggini (2020), a vitamina $\mathrm{C}$ pode ter efeitos benéficos em adultos e crianças com pneumonia. Bem como em pacientes em unidade de terapia intensiva (Hemilä \& Chalker, 2019). Mais de 100 estudos em animais indicaram que uma dose diária de algumas gramas de vitamina C pode aliviar ou prevenir infecções (Hemilä, 2017).

Um estudo preliminar, recente de pacientes com sepse e síndrome do desconforto respiratório agudo mostrou que a infusão por 96 horas de vitamina $\mathrm{C}$ em altas doses quando comparada ao placebo não proporcionou melhora significativa nos escores de disfunção orgânica ou alterou os marcadores de inflamação (Fowler, et al., 2019).

Em contrapartida, dados preliminares sugerem que a pneumonia, ocasionada devido a COVID-19 é uma complicação em estágio avançado causada pela hiperativação das células efetoras do sistema imunológico, e o tratamento com vitamina $\mathrm{C}$ em altas doses foi proposto para suprimir esses efetores (Erol, 2020).

Ocorre que o tratamento com vitamina C diminui a IL-6 e bloqueia in vivo a liberação de IL-6 no endotélio induzida pela endotelina-1 (ET-1) em humanos (Hemilä, Chalker, 2019; Bohm, Settergren, Pernow, 2007). O ET-1 é um potente peptídeo vasoconstritor, reconhecido também com citocina pró-inflamatória, sua expressão aumentada foi associada a pneumonia, hipertensão pulmonar, fibrose pulmonar intersticial e síndrome do desconforto respiratório agudo (Freeman, et al., 2014).

\subsection{Biodisponibilidade da vitamina $\mathrm{C}$}

A Biodisponibilidade inclui o modo como o composto bioativo atingiu a circulação sistêmica, como é transportado, como atinge o tecido alvo, a interação com o metabolismo das biomoléculas nesses tecidos e toda a cascata de efeitos fisiológicos que ocasiona (Cardoso, et al., 2015).

A vitamina $\mathrm{C}$ possui duas formas biologicamente ativas, ácido L-ascórbico e ácido L-desidroascórbico (Travica, et al., 2017), considerado um nutriente essencial para diversas funções metabólicas (Lykkesfeldt \& Tveden-Nyborg, 2019) e atuam como agente redutor ou antioxidante em muitas reações bioquímicas (Smirnoff, 2018).

O ácido ascórbico oxida rapidamente para a forma de ácido L-desidroascórbico, em pH neutro ou alcalino, e o ácido L-desidroascórbico oxida de forma não reversível para ácido 2,3-dicetogulônico (Pathy, 2018). Fatores como presença de oxigênio, luz, o esmagamento, atos como picar, lavar, cozinhar, enlatar e a presença de íons metálicos como cobre e ferro podem ocasionar perdas significativas de vitamina C nos alimentos (El-Ishaq \& Obirinakem, 2015).

A absorção intestinal de ácido ascórbico depende de transporte de sódio de transportador de vitamina C (SVTC1), enquanto a captação de ascorbato pelo cérebro depende do transportador SVTC2 (May, 2012; May, Nazarewicz \& Dikalov, 2013).

O desidroascorbato (DHA) é resultado da interação de dois radiais ascorbil (Du, Cullen \& Buettner, 2012), na forma extracelular pode ser captado por transportadores de glicose e, posteriormente, reduzido a ácido ascórbico por sistemas enzimáticos de tiol transferase citosólica. O DHA é instável em pH fisiológico e, se não for reduzido a ácido ascórbico, é prontamente degradado, gerando metabólitos que são eliminados na urina e, assim, esgotando as células de escorbuto (Du, Cullen \& Buettner, 2012). 


\section{Considerações Finais}

O consumo de quantidades adequadas de vitaminas e minerais através da alimentação é essencial para garantir o bom funcionamento do sistema imunológico, e foi demonstrado a estreita relação entre a imunidade e o consumo de vitamina $\mathrm{C}$, além dela ser responsável por reduzir tempo de internação e duração de ventilação mecânica em pacientes hospitalizados.

Foram evidenciados os efeitos positivos da vitamina $\mathrm{C}$ na melhora de sintomas em indivíduos acometidos com a COVID-19. Porém devido a fatores como perdas por processamento e o mecanismo de absorção que interferem na biodisponibilidade da vitamina no organismo, pode ser que haja necessidade da suplementação vitamínica para que ocorra os resultados esperados no organismo.

Por fim, levando em consideração o estudo realizado nota-se o ácido ascórbico atua no sistema imunológico, porém por ser estudos recentes ainda há uma escassez de dados que comprovem que a vitamina $\mathrm{C}$ atua como agente preventivo da COVID-19, desse modo, torna-se necessário a realização de mais estudos para uma maior exploração de dados.

\section{Referências}

Allegra, A., Tonacci, A., Pioggia, G., Musolino, C. \& Gangemi, S (2020). Vitamin deficiency as risk factor for SARS-CoV-2 infection: correlation with susceptibility and prognosis. European Review for Medical and Pharmacological Sciences, 24: 9721-9738.

Ang, A., Pullar, J. M., Currie, M. J., \& Vissers, M. C. M. (2018). Vitamin C and immune cell function in inflammation and cancer. Biochem Soc Trans. 46 (5), $1147-59$

Arabi, Y. M. \& Fowler. R. F. G (2020). Hayden Critical care management of adults with community-acquired severe respiratory viral infection. Intensive Care Med, 46: 315-328.

Barazzoni, R., Bischoff, S., Breda, J., Wickramasinghe, K., Krznaric, Z., Nitzan, D., Pirlich, M., \& Singer, P. (2020). Espen. experts statements and practical guidance for nutritional management of individuals with SARS- CoV2 infection. Clin Nutr. 39 (6), 1631-38.

Biasebetti, M. B. C., Rodrigues, I. D., \& Mazur, C. E. (2018). Relação do Consumo de Vitaminas e Minerais com o Sistema Imunitário: uma Breve Revisão. Visão Acadêmica, Curitiba, 191(1).

Bohm, F., Settergren, \& J. M. Pernow, (2007). Vitamin C blocks vascular dysfunction and release of interleukin-6 induced by endothelin-1 in humans in vivo. Atherosclerosis. 190, 408-415.

Calder, P. C., Carr, A. C., Gombart, A. F., \& Eggersdorfer, M. (2020). Optimal Nutritional Status for a Well-Functioning Immune System Is an Important Factor to Protect against Viral Infections. Nutrients, 12,4 1181.

Campos, L. F. et al (2020). Parecer BRASPEN/AMIB para enfrentamento do COVID 19 em pacientes Hospitalizados. BRASPEN J, 35(1), 3-5.

Carr, A. C., \& Maggini, S. (2017). Vitamin C and immune function. Nutrients, 9 p. 121.

Cavalari, T. G. F., \& Sanches, R. A (2018). Os Efeitos Da Vitamina C. Revista Saúde em Foco.

Dietary Reference Intakes (2000). Dietary reference intakes for vitamin C, vitamin E, selenium, and carotenoids. Washington, D. C. National Academy, 5, 95185 .

Dennis, J. M., \& Witting, P. K. (2017). Protective role for antioxidants in acute kidney disease. Nutrients, 9 , p. 718.

Dutra, A. de F. de O., Dias, A. D. C., Araújo, D. G. De S., et al. (2020). A importância da alimentação saudável e estado nutricional adequado frente a pandemia de Covid -19. Braz. J. of Develop., 6(9), 66464-66473.

El-Ishag, A., \& Obirinakem, S (2015). Effect of temperature and storage on vitamin C content in fruits juice. International Journal of Chemical and biomolecular Science, 1, 17-21.

Erol, A. (2020). High-dose intravenous vitamin C treatment for COVID-19. Erol Project Development House for the Disorders of Energy Megabolism, SilivriIstanbul, Turkey.

European Food Safety Authority (2013). Opinion on nutrient requirements and dietary intakes of infants and young children in the European Union. EFSA Journal, 11, 1-103.

Fowler, A. A., Truwit, J. D., Hite, R. D., Morris, P. E., DeWilde, C., Priday. A., et al (2019). Effect of vitamin C infusion on organ failure and biomarkers of inflammation and vascular injury in patients with sepsis and severe acute respiratory failure: The CITRIS-ALI randomized clinical trial. JAMA, 322, 12611270 .

Freeman, B. D., Machado F. S., Tanowitz, H. B., \& Desruisseaux, M. S. (2014). Endothelin-1 and its role in the pathogenesis of infectious diseases. Life Sci. 118, 110-119. 
Fuchs, F. D. (2010), Wannmacher L. Farmacologia Clínica: Fundamentos da Terapêutica Racional. (4a ed.), Guanabara Koogan.

Gombart, A. F., Pierre, A., \& Magginim, S. (2020). A review of micronutrients and the immune system-Working in harmony to reduce the risk of infection. Nutrients, 12, 236.

Grosso, G., Bei, R., Mistretta, A., Marventano, S., Calabrese, G., \& Masuelli, L (2013). Effects of vitamin C on health: a review of evidence. Front. Biosci. 18, $1017-1029$

Hemilä, H (2017). Vitamin C and Infections. Nutrientes. 9 (4):339. 10.3390/nu9040339. 28353648.

Hemilä, H., \& Chalker. E (2019). Vitamin C can shorten the length of stay in the ICU: A meta-analysis. Nutrientes, 11 (4), 708.

Hemilä, H., \& Chalker, E. (2020). Vitamin C may reduce the duration of mechanical ventilation in critically ill patients: a meta-regression analysis. $J$ Intensive Care. 8, 15.

Lai, C. C., Shih, T. P., Ko, W. C., Tang, H. J., \& Hsueh, P. R. (2020). Severe acute respiratory syndrome coronavirus 2 (SARS-CoV-2) and coronavirus disease-2019 (COVID-19): the epidemic and the challenges. Int. J. Antimicrob. Agents, 55, 105924.

Lei $\mathrm{n}^{\circ}$ 11.346, de 15 de setembro de (2006). Cria o Sistema Nacional de Segurança Alimentar e Nutricional - SISAN com vistas em assegurar o direito humano à alimentação adequada e dá outras providências. Diário Oficial da União.

Li, F. (2016). Structure, function and evolution of coronavirus spike proteins. Annu Rev Virol. 3, 237-61.

Lima, W. L., Batista, M. C. C., Silvino, V. O., Moura, R. C., Mendes, I. L., Moura, M. S. B., Batista, N. K. C., Silva, K. R., \& Barbosa, A. K. S. (2020). Importância nutricional das vitaminas e minerais na infecção da COVID-19. Research Society and Development, 9(8).

Marik, P. E. (2020). Vitamin C: An essential "stress hormone” during sepsis. J Thorac Disease, 12, S84-S88.

McEvoy, G. (2011). Drug Information. Bethesda, Maryland, USA: American Society of Health-System Pharmacists.

Mendes, E. N., Fiquene, J. C., Bessani, L., Santos Silva, F. C. M., Máximo, N. F., \& Alvarez, L. S. (2020). Hábitos de vida e consumo de alimentos pós pandemia de COVID-19 em São Luís, Maranhão, Brasil. Revista de Saúde Coletiva. Barueri, 10(58).

Murphy, K. (2019). Imunologia de Janeway. (8a ed.) Ed. Artmed. Nutrients, 11(708).

Pénicaud, C., Peyron, S., Bohuon, P., Gontard, N., \& Guillard, V. (2010). Ascorbic acid in food: Development of a rapid analysis technique and application to diffusivity determination. Food Research International, 43 (3), 838-847.

Pereira, S. A., et al (2018). Metodologia da pesquisa científica. UFSM. https://repositorio.ufsm.br/bitstream/handle/1/15824/Lic_Computacao_MetodologiaPesquisa-Cientifica.pdf?sequence $=1$.

Scavone, C., Brusco, S., Bertini, M., Sportiello, L., Rafaniello, C., Zoccoli, A., Berrino, G., Racagni F., \& Rossi, A (2020). Capuano Current pharmacological treatments for COVID-19: What's next? Br. J. Pharmacol., 177, 4813-4824.

Shakoor, H., Feehan, J., Ayesha S., Dhaheri, Al., Ali, H. I., Carine Platat, C., Ismail, C. L., Apostolopoulos, V., \& Stojanovska, L. (2021). Immune-boosting role of vitamins D, C, E, zinc, selenium and omega-3 fatty acids: Could they help against COVID-19? Maturitas, 143.

Siqueira, K. B., Binoti, M. L., Nunes, R. M., Borges, C. A. V., Pilati, A. F., Marcelino, G. W., Gama, M. A. S., \& Silva, P. H. F. (2020). Custo benefício dos nutrientes dos alimentos consumidos no Brasil. Revista de Ciências e Saúde Coletiva, 25(3), 1129-1135.

Spinola, V. A. R. (2011). Novas Metodologias para a Determinação do Conteúdo de Ácido Ascórbico em Alimentos Frescos. Dissertação De Mestrado em Bioquímica Aplicada. Universidade da Madeira. Portugal.

Tabela Brasileira de Composição de Alimentos (TACO) (2011). Coordenada pelo Núcleo de Estudos e Pesquisas em Alimentação (NEPA) da UNICAMP e com financiamento do Ministério da Saúde - MS e Ministério do Desenvolvimento Social e Combate à FOME.

Travica, N., Ried, K., Sali, A., Scholey, A., Hudson, I., \& Pipingas, A (2017). Vitamin C status and cognitive function: A systematic review. Nutrients. 9(9):960. 10.3390/nu9090960.

Wang, Y., Wang, Y., Chen, Y., \& Qin, Q. (2020). Unique epidemiological and clinical features of the emerging 2019 novel coronavirus pneumonia (COVID19) implicate special control measures. J. Med. Virol. 92, 568-576.

Zeng, Y. C., Cai, Z. X., Xianyu, Y. Y., Yang, B. X., Ting, C., \& Yan, O (2020). Prognosis when using extracorporeal membrane oxygenation (ECMO) for critically ill COVID-19 patients in China: a retrospective case series. Crit Care, 24, 148. 